\title{
Knock-down Nanog gene expression by siRNA affects the differentiation of cardiomyocytes in P19 cell
}

\author{
Lei Lei ${ }^{1}$, Lin Dou ${ }^{1}$, Huayan Wang ${ }^{1}$ \\ ${ }^{1}$ Shaanxi Branch of National Stem Cell Engineering \& Technology Center, Northwest A\&F University, Yangling, Shaanxi 712100, \\ China
}

Aim: Nanog is a transcriptional factor and plays a key role to maintain embryonic stem (ES) cell self-renew and pluripotency. Down regulating nanog gene expression will initiate ES cell differentiation. This study was designed to inhibit nanog gene expression by novel siRNA fragment and analysis its effect on the embryoid body formation and cardiomyocytes differentiation in P19 cells.

Methods: Based on the published mouse nanog cDNA sequence, two primers which were designed to amplify a 912 bp nanog cDNA and three siRNA fragments were synthesized. The details are listed below:

bnanogS: ATAGATCTCTGAGTGGGTCTTCCTGGTC;

bnanogA: TATCCGCGGTCATATTTCTCCTGGTG;

N301: AAGCAGAAGAUGCGGACUGUGUU (XM_132755, 301 323 bp);

N745: AAUGCUGCUCCGCUCCAUAACUU (XM_132755, 745 768 bp);

N849: UAGGGAAAGCCAUGCGCAUUUUA (XM_132755, 849 872 bp);

NC: UUCUCCGAACGUGUCACGUTT

In addition of three siRNA fragments that were designed to inhibit nanog expression in ES and P19 cells, one unspecific siRNA fragment was also synthesized as the negative control. siRNA fragments were transferred with Lipofectamine 2000 into ES-129 and P19 cells. After 24 h siRNA transfection, total RNA was isolated from cells by TriZol method. To form the embryoid body, P19 $\left(1 \times 10^{4} / \mathrm{ml}\right)$ were grown by suspending cells in hanging drops for 48 $h$. The differentiation of cardiomyocyte was facilitated by the formation of aggregates after adding $1 \%$ DMSO into culture medium. After 2 days growth, the induced embryoid bodies were cultured on tissue culture plate for $\mathbf{4}$ more days, and the rhythmic beating cardiomyocytes were observed.

Results: To investigate the inhibition efficiency, three siRNA fragments were transfected in ES cells, respectively. After $24 \mathrm{~h}$ transfection, the expression of nanog gene was analyzed by RT-PCR. N849 reduced the level of Nanog gene expression down $30 \%$, while $\mathbf{N} 745$ only slightly altered Nanog expression. The result showed that $\mathrm{N} 301$ significantly decreased the level of Nanog gene expression in 90\% comparing with the control. ES cells transfected with N301 for $24 \mathrm{~h}$ started to differentiate and to gradually lose typical ES morphology within the $48 \mathrm{~h}$ treatment. The similar results were also observed in P19 cells. The results showed that down regulation of Nanog activity interfered P19 cells to form embryoid bodies and reduce the capability to differentiate into cardiomyocytes.

Conclusion: The results showed that N301 siRNA was efficiently interfered in nanog gene expression in both mouse ES and P19 cells. Down regulation of nanog gene expression initiated ES cell differentiation and lowered the ability of $P 19$ cell to form embryoid bodies.

Keywords: Nanog, siRNA, ES cells, P19, RT-PCR

Cell Research (2008) 18:s36. doi: 10.1038/cr.2008.126; published online 4 August 2008

Correspondence: Huayan Wang

E-mail: hhwang101@163.com 